\title{
Kann man den Eisprung hören?
}

\section{Bisher wurde vermutet, dass die Stimmlage einer Frau den Eisprung verrät. Das stimmt aber so wohl doch nicht, wie Forscher in Göppingen jetzt herausfanden.}

- Um sicher zu sein, dass die Stimmbänder der 18- bis 26-jährigen Frauen, die an der Studie teilnahmen, vergleichbar waren, untersuchte ein Arzt die Stimmbänder und den Kehlkopf. Raucherinnen, Asthmatikerinnen, Sängerinnen, Moderatorinnen oder Frauen, die ihre Stimme stark beanspruchten, durften nicht teilnehmen. Während des gesamten Zyklus wurde der Hormonspiegel im Urin gemessen, um den genauen Zeitpunkt des Eisprungs zu ermitteln. Zusätzlich zeichneten die Frauen ihre Stimmen auf: Sie sprachen eine Minute lang über das Wetter oder erzählten vom vergangenen Tag. Zusätzlich wurden die Vokale „A“, „U“ und „I“ aufgenommen, weil sie sich am deutlichsten voneinander unterscheiden.

Je näher der Eisprung rückte, desto höher wurden die Stimmen. Doch am Tag des Eisprungs selbst senkte sich bei 17 von 23 Probandinnen die Tonhöhe um durchschnittlich sechs Hertz bei den Vokalen und zehn Hertz bei der freien Sprache. Nach wenigen Tagen kehrte sie jedoch wieder zur höheren Stimmlage zurück.

Um zu sehen, wie Männer auf die Stimmlagen der Frauen während der unterschiedlichen Zyklusphasen reagieren, spielten die Forscher 28 Männern Aufnahmen mit den Stimmen vor, während und nach dem Eisprung vor. Die Probanden sollten entscheiden, welche der drei Stimmen am attraktivsten klingt. Lediglich 15 von 28 zogen die Stimmen kurz vor dem Eisprung den anderen vor.

\section{Kommentar}

Frühere Studien hatten die Frauenstimmen lediglich zum Zeitpunkt der höchsten und der niedrigsten Fruchtbarkeit untersucht. Nun sahen sich die Wissenschaftler den gesamten Verlauf an und machten deutlich, dass die Prozesse, die zur Fortpflanzung anregen, komplexer sind. Die Stimme einer Frau signalisiert den Zeitpunkt des Eisprungs nicht, obwohl die Sexualhormone die Tonlage beeinflussen. Schwankungen der Sexualhormone Östrogen und Progesteron wirken sich zwar indirekt auf die Tonfrequenzen aus, beeinflussen diese aber nicht so stark, dass Männer den Frauen unterbewusst beim Fruchtbarwerden „zuhören“ könnten.

K. MALBERG =

\section{- J. Fischer et al.}

Do women's voices provide cues of the likelihood of ovulation? The importance of sampling regime. Published on September 21, 2011 in PlosONE 6; 2011, e 24490; doi: PLoS ONE 6(9): e24490. doi:10.1371/journal.pone.0024490

\section{Darm-Rückzug durch Zucker}

Etwa zwei Jahre nach Anlage eines lleostomas wegen einer Mesenterialarterienembolie stellte sich ein 62-jähriger Mann mit einem ausgeprägten Prolaps des Ileums in der Nothilfe vor (Abb. links). Er hatte den Prolaps etwa zwölf Stunden vor der Vorstellung bemerkt. Der Versuch einer manuellen Reposition war nicht erfolgreich.
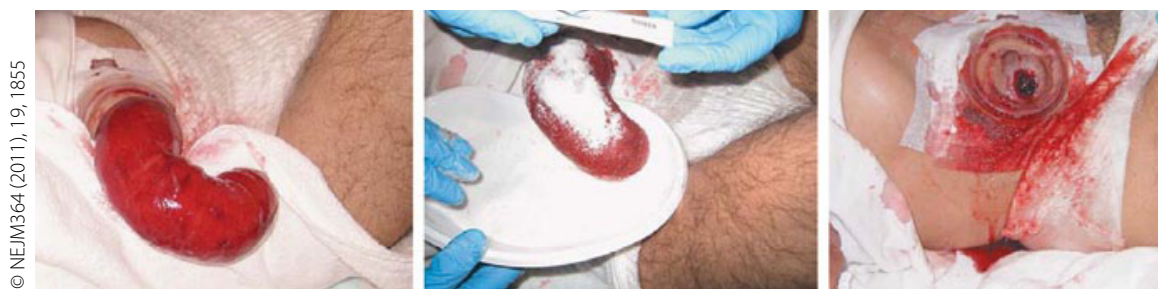

Bei Tierärzten abgeschaut: Der Prolaps wird mit Zucker rückgängig gemacht.

— Aufgrund der kardiopulmonalen Erkrankungen des Patienten wurde ein chirurgischer Eingriff in Vollnarkose als sehr riskant angesehen. Daher entschloss man sich zu einer ungewöhnlichen Maßnahme. In Analogie zum konservativen Management von Analprolapsen und Uterusprolapsen bei Rin- dern wurde auf die Mukosa des Ileums einfacher Zucker gestreut, um so einen osmotischen Flüssigkeitsentzug aus dem ödematösen Gewebe zu bewerkstelligen (Abb. Mitte). Innerhalb von zwei Minuten war das Schleimhautödem soweit zurückgegangen, dass sich der Prolaps spontan reponierte (Abb. rechts).
24 Sunden später zeigte sich bei einer endoskopischen Untersuchung eine leichte Ischämie in den distalen Anteilen des Ileums, die aber keine weitere Behandlungsmaßnahmen erforderte. Der Patient wurde am folgenden Tag entlassen. Auch im Lauf der folgenden sechs Monate kam es nicht zu einem weiteren Prolaps.

\section{Kommentar}

Ungewöhnliche Situationen erfordern manchmal ungewöhnliche Maßnahmen. Wohl dem, der über gute Kontakte zu anderen Disziplinen verfügt - und seien es veterinärmedizinische.

H. S. FÜESSL .

- A. R. M. L. Brandt, O. Schouten

(Reinier de Graaf Hospital, Delft o.schouten@ erasmusms.nl): Sugar to reduce a prolapsed ileostomy. New Engl. J. Med. 364 (2011), 19, 1855 